\title{
A Paradigm Shift to Align Transmission Routes with Mechanisms
}

\author{
Linsey C. Marr, ${ }^{a}$ Ph.D. and Julian W. Tang, ${ }^{b}$ Ph.D., F.R.C.Path \\ ${ }^{a}$ Civil and Environmental Engineering, Virginia Tech, Blacksburg, VA, USA \\ ${ }^{\mathrm{b}}$ Respiratory Sciences, University of Leicester, Leicester, UK
}

Corresponding author: 1145 Perry St. (0246), Durham 411, Blacksburg, VA, 24060 USA; lmarr@vt.edu; 540-231-6071 (phone); 540-231-7916 (fax)

Alternate contact: University Hospitals of Leicester National Health Service Trust, Clinical Microbiology and Virology, Level 5 Sandringham Bldg, Leicester Royal Infirmary, Infirmary Square, Leicester LE1 5WW, UK; jwtang49@ hotmail.com

Keywords: transmission, airborne, aerosols, droplets, respiratory

Running title: Transmission routes 


\begin{abstract}
Current infection control guidelines subscribe to a contact/droplet/airborne paradigm that is based on outdated understanding. Here, we propose to modify and align existing guidelines with a more accurate description of the different transmission routes. This will improve the effectiveness of control measures as more transmissible variants of SARS-CoV-2 emerge.
\end{abstract}




\section{Commentary}

With the relaxation of various non-pharmaceutical restrictions (masking and social distancing), and the opening of international borders to travel again, we need to prepare longer-term strategies for how we manage COVID-19 as it becomes more seasonal [1]. Highly transmissible SARSCoV-2 variants, such as Delta (B.1.617.2), are rapidly spreading around the world, escaping complete vaccine protection [2-4], as well as transmitting quickly through unvaccinated and partially vaccinated populations. Hence, non-vaccine control strategies remain equally important. These can only be effective, however, if they target the main route(s) of transmission. Therefore, a correct and thorough understanding of how SARS-CoV-2 transmits underpins the design and implementation of effective control strategies. Just as importantly, it reduces the time, effort, and resources wasted on ineffective actions (i.e., "hygiene theater").

The pandemic has revealed inconsistencies and inaccuracies in the traditional understanding of transmission of respiratory viruses. This misunderstanding, entwined with operational definitions for infection control, has led to confusion and disagreement. As a result, public health agencies were slow to recognize the importance of transmission of SARS-Cov-2 by aerosols, communication with the general public failed to emphasize the most effective interventions, and the actual mechanisms by which pathogens move through the environment while transmitting person-to-person have become obscured. Addressing these limitations is crucial to examining the ongoing response to the pandemic, as SARS-CoV-2 becomes more seasonal, as well as preparing for the next one.

In the same context, it is important to understand how and why such erroneous concepts and beliefs arose, in order to correct and update them more effectively. Traditional routes of transmission were established in the early $20^{\text {th }}$ century as (1) direct contact with an infected person or indirect contact via a contaminated object known as a "fomite," (2) droplets propelled in a cough or sneeze by an infected person onto the mucous membranes of a susceptible person, and (3) droplet nuclei or aerosols, consisting of dried residue from droplets that have evaporated [5, 6], that transmit disease over long distances. The latter is known as "airborne" transmission and became associated with particles smaller than $5 \mu \mathrm{m}$ [7]. 
While these classifications seem like a reasonable way to think about transmission mechanisms, there are two main problems with them.

First, they introduce technical inaccuracies that defy physics and reflect an incomplete understanding of emissions during respiratory activities. This has led to incorrect assumptions and a misleading mental model about how pathogens actually move through the environment and reach a susceptible individual. According to this model, droplets larger than $5 \mu \mathrm{m}$ follow ballistic trajectories (i.e., like when a ball is thrown) and travel a limited distance before falling to the ground. Droplets were believed to be solely responsible for transmission at close range $(<1-2$ meters). In contrast, aerosols were believed to be only of significance over longer ranges. Droplets and aerosols were thought to be produced mainly by coughing and sneezing or by aerosol generating medical procedures.

While previous publications, including our own, have attempted to separate droplets and aerosols by various criteria, here we start with a clean slate and build on widely-accepted definitions that are not encumbered by discipline-specific connotations. According to dictionaries, a droplet is "a small drop of a liquid"; no minimum or maximum size is specified. Thus, all bits of fluid that are released from the respiratory tract can be considered to be droplets initially. Depending on ambient humidity, they may partially or fully evaporate, shrinking to $20-40 \%$ of their initial diameter, in which case they may be solid or semi-solid [8]. According to dictionaries, an aerosol is "a suspension of fine solid particles or liquid droplets in a gas." Aerosol particles, whether solid or liquid, are often referred to simply as "aerosols." We will refer to droplets that are large enough to fall to the ground within a few seconds, and that do not evaporate significantly within this time period, as "large droplets." Based on these definitions, the threshold between aerosols and large droplets is approximately $100 \mu \mathrm{m}$ (the average diameter of a human hair) and depends on environmental conditions such as humidity and air flow velocity and direction [9-11]. This threshold is also the upper limit of particles that can be inhaled [12, 13].

Using this terminology, we can now describe the transfer of pathogens in respiratory fluid from one person to another. People produce respiratory droplets spanning a wide range of sizes, from submicron to millimeter, when breathing, talking, singing, coughing, and sneezing. The size distribution is multimodal with a smaller mode around 1-3 $\mu \mathrm{m}$, thought to originate in the 
bronchioles and larynx, and a larger mode around 100-200 $\mu \mathrm{m}$, thought to originate in the oral region $[14,15]$. The smaller ones are far more numerous than the larger ones, and the majority of any given pathogen is typically found in the smaller ones, even though the larger ones contain the majority of the volume of fluid [16]. Droplets are emitted as part of a turbulent cloud. After a few seconds, the initial respiratory jet slows down and merges with the background air, and the droplets then behave as individual entities $[17,18]$.

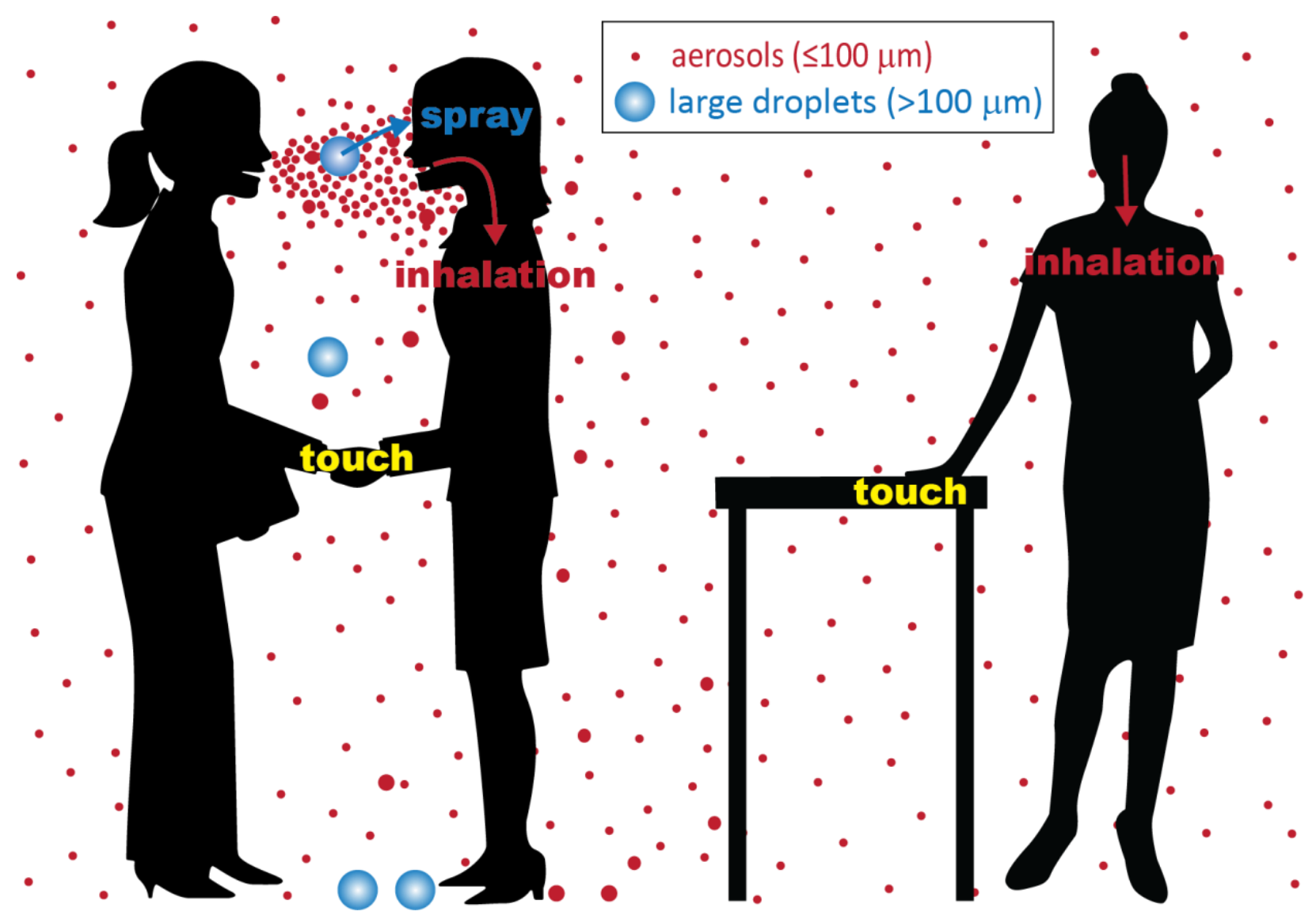

Figure 1. Schematic of an infected person talking and releasing respiratory droplets in a poorly ventilated room, where small aerosols can mix and accumulate throughout the space. Transmission routes are based on how pathogens move through the environment and how people are exposed to them: inhalation, spray, and touch. At close range, all routes are possible, whereas at a distance, transmission only by inhalation and touch of a previously contaminated surface are possible. 
The second problem is that the three traditional transmission routes became entangled with and limited by the various operational definitions for the precautions required for infection prevention and control of different diseases. According to these operational practices, COVID-19 must be classified as either a "droplet" or "airborne" disease, where the latter has been thought to be relevant mainly at long distances. This dichotomy overlooks the reality that respiratory droplets of all sizes, including aerosols, are most concentrated close to the source (i.e., the infected individual) and that exposure at all but uncomfortably close distances is dominated by inhalation rather than the impaction of large droplets that are sprayed onto mucous membranes [19, 20]. Trying to impose this dichotomy and operational definitions on the actual mechanisms of transmission has contributed to incredibly confusing and inconsistent communication about how such pathogens actually behave in the environment and how people can best protect themselves.

To overcome these limitations, we propose a shift in how we define transmission routes, to better align them with the best available evidence for actual mechanisms of transmission. This framework will be more accurate and more truly representative of how pathogens move through the environment, and therefore how people are exposed to them.

Based on decades of multidisciplinary research on transmission mechanisms, a US National Academies of Sciences, Engineering, and Medicine workshop on transmission of SARS-CoV-2 [21], and publications by longtime researchers on the topic [12, 13, 22-24], we propose three major routes of transmission (Figure 1b):

1) Inhalation. Pathogens carried by aerosols can be directly inhaled into the respiratory tract and deposited at various sites, depending on the size of the aerosol. Aerosols are most concentrated close to the point of release (an infected person), and the smaller ones can remain floating in the air for minutes to hours and can be carried long distances on local air currents. Transmission can occur at any distance and is more likely when people are in close proximity.

2) Spray. Pathogens in large droplets may land directly onto external mucous membranes. Large droplets follow semi-ballistic trajectories [25] and settle to the ground within a few seconds. Usually, they do not travel farther than 1-2 meters, although a sneeze can propel them farther [26]. 
3) Touch. A susceptible individual transfers a pathogen, usually by hand, to their mucous membranes. This may occur, for example, if the individual shakes hands with an infected person who wiped their nose or touches a contaminated object.

These definitions directly correspond to different interventions: respirators and masks with excellent fit and filtration capability, along with ventilation and filtration to reduce transmission by inhalation; distancing and face shields to mitigate transmission by sprays (where surgical masks and face coverings are also effective to some degree); and hand hygiene, surface cleaning, and gloves to reduce transmission by touch.

An accurate understanding of transmission reduces the fear associated with it and informs and empowers people to select and plan for more effective interventions. We believe that aligning how we talk about different transmission routes, particularly within an infection control context, with actual mechanisms of transmission, will lead to clearer communication and an improved understanding of and compliance with public health guidance.

\section{Funding}

None

\section{Acknowledgments}

The authors thank John Brooks, Lisa Brosseau, Yuguo Li, Matthew Meselson, Don Milton, Ronke

Olabisi, Kim Prather, and the two reviewers for helpful discussions and feedback. Artwork by Jasper Marr Hester.

\section{References}

1. Murray CJL, Piot P. The potential future of the COVID-19 pandemic: will SARS-CoV-2 become a recurrent seasonal infection? JAMA 2021; 325(13): 1249-50. 
2. Sheikh A, McMenamin J, Taylor B, Robertson C. SARS-CoV-2 Delta VOC in Scotland: demographics, risk of hospital admission, and vaccine effectiveness. The Lancet 2021; 397(10293): 2461-2.

3. Wall EC, Wu M, Harvey R, et al. Neutralising antibody activity against SARS-CoV-2 VOCs B.1.617.2 and B.1.351 by BNT162b2 vaccination. The Lancet 2021; 397(10292): 2331-3.

4. Wall EC, Wu M, Harvey R, et al. AZD1222-induced neutralising antibody activity against SARS-CoV-2 Delta VOC. The Lancet 2021; 398(10296): 207-9.

5. Chapin C. The Sources and Modes of Infection. New York: John Wiley \& Sons, Inc., 1916.

6. Wells WF. On air-borne infection. Study II. Droplets and droplet nuclei. American Journal of Epidemiology 1934; 20(3): 611-8.

7. Zhang N, Chen W, Chan P-T, Yen H-L, Tang JW-T, Li Y. Close contact behavior in indoor environment and transmission of respiratory infection. Indoor Air 2020; 30(4): 645-61.

8. Marr LC, Tang JW, Van Mullekom J, Lakdawala SS. Mechanistic insights into the effect of humidity on airborne influenza virus survival, transmission and incidence. Journal of The Royal Society Interface 2019; 16(150): 20180298.

9. Liu L, Wei J, Li Y, Ooi A. Evaporation and dispersion of respiratory droplets from coughing. Indoor Air 2017; 27(1): 179-90.

10. Xie X, Li Y, Chwang ATY, Ho PL, Seto WH. How far droplets can move in indoor environments - revisiting the Wells evaporation-falling curve. Indoor Air 2007; 17: 21125 .

11. Prather KA, Wang CC, Schooley RT. Reducing transmission of SARS-CoV-2. Science 2020: eabc6197.

12. Milton DK. A Rosetta stone for understanding infectious drops and aerosols. Journal of the Pediatric Infectious Diseases Society 2020; 9(4): 413-5.

13. Jones RM, Brosseau LM. Aerosol transmission of infectious disease. Journal of Occupational and Environmental Medicine 2015; 57(5): 501-8.

14. Johnson GR, Morawska L, Ristovski ZD, et al. Modality of human expired aerosol size distributions. Journal of Aerosol Science 2011; 42(12): 839-51.

15. Morawska L, Johnson GR, Ristovski ZD, et al. Size distribution and sites of origin of droplets expelled from the human respiratory tract during expiratory activities J Aerosol Sci 2009; 40: 257-69.

16. Fennelly KP. Particle sizes of infectious aerosols: implications for infection control. The Lancet Respiratory Medicine 2020. 
17. Bourouiba L. Fluid dynamics of respiratory infectious diseases. Annual Reviews in Biomedical Engineering 2021; 23: 547-77.

18. Li Y. The respiratory infection inhalation route continuum. Indoor Air 2021; 31(2): 27981.

19. Chen W, Zhang N, Wei J, Yen H-L, Li Y. Short-range airborne route dominates exposure of respiratory infection during close contact. Building and Environment 2020; 176: 106859.

20. Cortellessa G, Stabile L, Arpino F, et al. Close proximity risk assessment for SARS-CoV2 infection. Science of The Total Environment 2021; 794: 148749.

21. Samet JM, Prather K, Benjamin G, et al. Airborne transmission of SARS-CoV-2: what we know. Clinical Infectious Diseases 2021.

22. Li Y. Basic routes of transmission of respiratory pathogens-A new proposal for transmission categorization based on respiratory spray, inhalation, and touch. Indoor Air 2021; 31(1): 3-6.

23. Tang JW, Marr LC, Milton DK. Aerosols should not be defined by distance travelled. Journal of Hospital Infection 2021.

24. Tang JW, Marr LC, Li Y, Dancer SJ. Covid-19 has redefined airborne transmission. BMJ 2021; 373: n913.

25. Bourouiba L. Turbulent gas clouds and respiratory pathogen emissions: potential implications for reducing transmission of COVID-19. JAMA 2020; 323(18): 1837-8.

26. Bourouiba L, Dehandschoewercker E, Bush John WM. Violent expiratory events: on coughing and sneezing. Journal of Fluid Mechanics 2014; 745: 537-63. 


\section{Figure Caption}

Figure 1. Schematic of an infected person talking and releasing respiratory droplets in a poorly ventilated room, where small aerosols can mix and accumulate throughout the space. Transmission routes are based on how pathogens move through the environment and how people are exposed to them: inhalation, spray, and touch. At close range, all routes are possible, whereas at a distance, transmission only by inhalation and touch of a previously contaminated surface are possible. 